\title{
Caracterización de la gestión de residuos hospitalarios y similares en CAMI Vista Hermosa, Bogotá
}

\author{
Characterization of hospital and similar waste management in CAMI Vista Hermosa, \\ Bogota
}

\section{Caracterização do gerenciamento de resíduos hospitalares e similares no CAMI Vista Hermosa, Bogotá}

Juan Carlos Hernández-Criado ${ }^{1}$

Forma de citar: J. C. Hernández-Criado, "Caracterización de la gestión de residuos hospitalarios y similares en CAMI Vista Hermosa, Bogotá”, Respuestas, vol. 21, no. 1, pp. 6-15, 2016.

Recibido:

Mayo 19 de 2015

Aceptado:

Septiembre 30 de 2015

${ }^{1}$ Magister Desarrollo Sustentable y Gestión Ambiental jchernandezc@ufpso.edu.co. Orcid: 0000-0002-4591-4047 Universidad Francisco de Paula Santander Ocaña-Colombia.

\section{Resumen}

Antecedentes. La gestión ambiental de los residuos, se enmarca en la política pública, la cual involucra los residuos de carácter peligroso de una forma generalizada. Solo hasta el año 2000, se establecen los lineamientos reglamentarios para la gestión integral de los residuos hospitalarios y similares en Colombia. Objetivo. Caracterizar la gestión de residuos hospitalarios y similares en CAMI VISTA HERMOSA Bogotá. Métodos. La investigación se desarrolló en la Institución Prestadora de Servicios de Salud de primer nivel CAMI VISTA HERMOSA, correspondiente a una Empresa Social del Estado. Se desarrolló un estudio tipo corte transversal, donde se caracterizaron cuantitativa y cualitativamente los residuos generados por día, a través de la consolidación de la información en el formato RH1, establecido por el Manual para la Gestión Integral de los Residuos Sólidos Hospitalarios y Similares. Resultados. Se identificaron residuos como, Biosanitarios, Cortopunzantes, Fármacos, Metales Pesados, Ordinarios, Reciclables y Anatomopatológicos por medio del formato RH1, así mismo fueron cuantificados en cada una de las áreas involucradas en el proceso de prestación del servicio, mostrando la fluctuación en el comportamiento referente a pesos y tipos de estos, determinados por distintos factores, entre los que se tienen: la creciente asistencia a los servicios por consultas de enfermedades respiratorias, asociadas a las temporadas de lluvia y por la inadecuada segregación de los residuos en distinta áreas. Conclusiones. La recolección de la información, sirvió como herramienta para identificar los puntos críticos, donde se realiza una segregación inadecuada de los residuos. Información que además fue útil como estrategia para reorganizar los procesos de cambio desde la óptica del Plan de Gestión de Residuos Hospitalarios y Similares.

Palabras Clave: Bioseguridad, Centro de Atención Medico Integral (CAMI), Gestión de Residuos, Impacto Ambiental, Salud Pública.
Abstract
Background. The environmental management of residues is defined within the public policy, which involves the hazardous waste from a general standpoint. Only until 2000, the mandatory guidelines were established for the comprehensive management of hospital waste and similar in Colombia. Objective. To characterize the management of hospital 
and similar waste in CAMI VISTA HERMOSA, Bogotá. Methods. Research was performed at the first level healthcare provider CAMI VISTA HERMOSA, a public social company. A cross - section type study was performed, where the daily-base generated waste was characterized qualitatively and quantitatively, through the consolidation of the information in the RH1 form, established by the Manual for the Comprehensive Management of Hospital Solid Waste and Similar. Results. Biosanitary, sharps, drug, heavy metal, ordinary, recyclable and anatomic-pathological wastes were identified by using the RH1 form; similarly, they were quantified in each of the areas involved in the service providing process, showing the fluctuation in the behavior related to weights and each of their classes, determined by several factors including: the increasing number of medical appointments regarding respiratory diseases associated with raining seasons and the inappropriate waste disposal in different areas. Conclusions. Data collection was a tool that allowed to identify critical points such as the inappropriate waste disposal. Besides, the information was useful as a strategy to reorganize processes of change according to the Plan of Management of Hospital Waste and Similar.

Keywords: Biosafety, Waste Management, Environmental Impact, Public Health.

\section{Resumo}

Antecedentes. A gestão ambiental dos resíduos faz parte da política pública, a qual involucra os resíduos de carácter perigoso de uma forma generalizada. Apenas até 2000, se estabelecem os lineamentos regulamentários para a gestão integral dos resíduos hospitalares e similares na Colômbia. Objetivo. Caracterizar a gestão de resíduos hospitalares e similares no CAMI VISTA HERMOSA Bogotá. Métodos. A pesquisa se desenvolveu na Instituição Oferente de Serviços de Saúde de primeiro nível CAMI VISTA HERMOSA, correspondente a uma Empresa Social do Estado. Desenvolveu-se um estudo tipo corte transversal, onde se caracterizaram quantitativa e qualitativamente os resíduos gerados por dia, através da consolidação das informações no formato RH1, estabelecido pelo Manual para a Gestão Integral dos Resíduos Sólidos Hospitalares e Similares. Resultados. Identificaram-se resíduos tais como: Biosanitarios, Perfurocortantes, Medicamentos, Metais Pesados, Ordinários, Recicláveis e Anatomopatológicos por médio do formato RH1, assim mesmo foram quantificados em cada uma das áreas involucradas no processo de prestação do serviço, mostrando a flutuação no comportamento referente a pesos e tipos destes, determinados por diferentes fatores, entre eles: a crescente assistência aos serviços por consultas de doenças respiratórias, associadas às temporadas de chuva e pela inadequada segregação dos resíduos em diferentes áreas. Conclusões. A recolecção da informação serviu como ferramenta para identificar os pontos críticos, onde se realiza uma segregação inadequada dos resíduos. Esta informação também foi útil como estratégia para reorganizar os processos de mudança desde a óptica do Plano de Gerenciamento de Resíduos Hospitalares e Similares.

Palavras-chave: Biossegurança, Centro de Atenção Medico Integral (CAMI), Gerenciamento de Resíduos, Impacto Ambiental, Saúde Pública. 
No. 1

Enero - Junio 2016 ISSN 0122-820X E-ISSN 2422-5053 PP: 6-15

\section{Introducción}

Los residuos hospitalarios y similares son sustancias de carácter peligroso en estado líquido, solido o gaseoso generados por una actividad en servicios asistenciales en salud [1]. En Colombia en las Instituciones Prestadoras de Salud (IPS) del estado niveles I, II y III se producen entre 5560 y 8500 toneladas de residuos hospitalarios y similares por año, el $40 \%$ de estos es de carácter infeccioso y el $60 \%$ restante corresponde a material contaminante que podría aumentar el riesgo de enfermedades e impactos a la salud pública [2].

Pese a que la legislación ha procurado que en Colombia se realice a través de los planes de gestión de residuos hospitalarios y similares su adecuado manejo y disposición, las entidades del sector salud involucradas han tenido problemas para el cumplimiento de la normativa debido a la falta de capacitación del recurso humano, deficiencias en la compra, manejo de insumos, su almacenamiento y disposición final, falta de rutas internas y la inadecuada segregación en la fuente [3],[4].

Los residuos generados por una comunidad requieren de un sistema de tratamiento y disposición final. El proceso correspondiente a la gestión de los residuos hospitalarios, requiere una estrategia desde la generación hasta la disposición final del residuo, los cuales son causantes de una gran variedad de impactos al medio ambiente y a la salud [5]. Uno de los mecanismos de mayor relevancia y preocupación en la gestión de los residuos hospitalarios, es la gestión a los residuos de carácter peligroso o infeccioso generados por los procesos productivos asistenciales, los cuales representan una problemática de gran impacto a los recursos naturales y por ende el de mayor relevancia a la salud [6]. Es así como el presente estudio adquiere su relevancia debido a que los residuos generados en la IPS CAMI VISTA HERMOSA, son considerados peligrosos.
Diariamente se genera la transformación inminente del territorio, donde el factor de mayor incidencia es el crecimiento demográfico, en el que las tendencias de uso de recursos y consumistas, van aumentando con el tiempo. Estos cambios modifican las condiciones normales de los recursos suelo, agua y aire [7], determinando una gran variedad de estrategias, las cuales traen consigo un incremento en el desarrollo tecnológico y los procesos de desarrollo industrial, transformando los patrones de consumo de las poblaciones e impactando los procesos ambientales y la salud pública. De esta manera es relevante establecer la problemática que se genera en el marco de la atención de los servicios de salud, factor transformador del medio.

El manejo inadecuado de los residuos hospitalarios representa un riesgo para las personas y el medio ambiente, por la presencia de residuos infecciosos $o$ de riegos biológico, tóxicos, químicos y objetos cortopunzantes [8]-[10]. Dichos residuos pueden generar enfermedades parasitarias, infecciosas como: el SIDA, la Hepatitis B y $\mathrm{C}$, infecciones gastroenteriticas, infecciones respiratorias, infecciones dérmicas e intoxicaciones, causadas por microrganismos como Pseudomonas aeruginosa, Klebsiella sp, Enterobacter sp [2], [7], las cuales representan un riesgo potencial para la salud del personal asistencial como enfermeras, médicos, personal operativo de servicios generales y aseo, usuarios y personal externo que manipulan los residuos en el proceso de tratamiento, transporte y disposición final, que tenga contacto directo o no con estos, así como los responsables de su gestión, la comunidad hospitalaria, la población en general y el medioambiente.

El objetivo de esta investigación, fue determinar la producción cualitativa y cuantitativa de los residuos hospitalarios $\mathrm{y}$ similares en la IPS CAMI VISTA 
HERMOSA, en búsqueda del cumplimiento de la normatividad vigente expedida por los Ministerios de Medio Ambiente y Salud, Decreto 2676 de 2000 y Resolución 1164 de 2002, con el fin de establecer las cantidades de residuos de tipo peligroso que se generaron en el centro asistencial para un periodo de tiempo de 12 meses y de esta manera determinar estrategias para minimizar las cantidades totales y la forma de clasificación, lo que colabore para evitar la propagación de enfermedades infecciosas, el aumento en la cantidad de desechos, la reducción de los costos del servicio de tratamiento, la disposición final y el reconocimiento de los costos ambientales que implica la gestión ambiental de los desechos hospitalarios.

\section{Materiales y métodos}

Se realizó un estudio de corte transversal, durante doce meses, en las instalaciones del centro asistencial CAMI VISTA HERMOSA, perteneciente a la Red Hospitalaria Pública de Bogotá Colombia (Figura 1), I Nivel de atención, como proceso de control cuantitativo y cualitativo a los residuos generados en esta. El centro asistencial, se encuentra ubicado en la Localidad 19 Ciudad Bolívar al sur de Bogotá D.C. Colombia, el cual presta los servicios las 24 horas del día en Urgencias,
Atención de Partos, Hospitalización de Adultos y Pediátrico, Apoyo Diagnóstico, Laboratorio Clínico, Toma de Muestras de Laboratorio, Rayos X, Ecografías Obstétricas, Medicina General, Enfermería, Odontología, Salas de Enfermedad Respiratorias Agudas y Vacunación, los cuales generan en sus servicios una serie de residuos de carácter peligroso.

\subsection{Muestreo}

El tipo de muestreo aplicado fue aleatorio estratificado, donde se tomó como población los residuos hospitalarios y similares, la cual se dividió en grupos de residuos con características particulares. Se realizó una jornada de caracterización para un tiempo de 12 meses, que sí bien es un período suficiente para determinar datos promedios de generación a nivel institucional, nos ayudó a verificar como se está realizando la labor de segregación, recolección, almacenamiento y pesaje de residuos con el apoyo de una báscula tipo gramera digital, lo cual contribuyó a generar un primer dato de producción de residuos por área o servicio.

En esta investigación se determinó como estrategia principal la observación directa y el registro mensual consolidado del peso

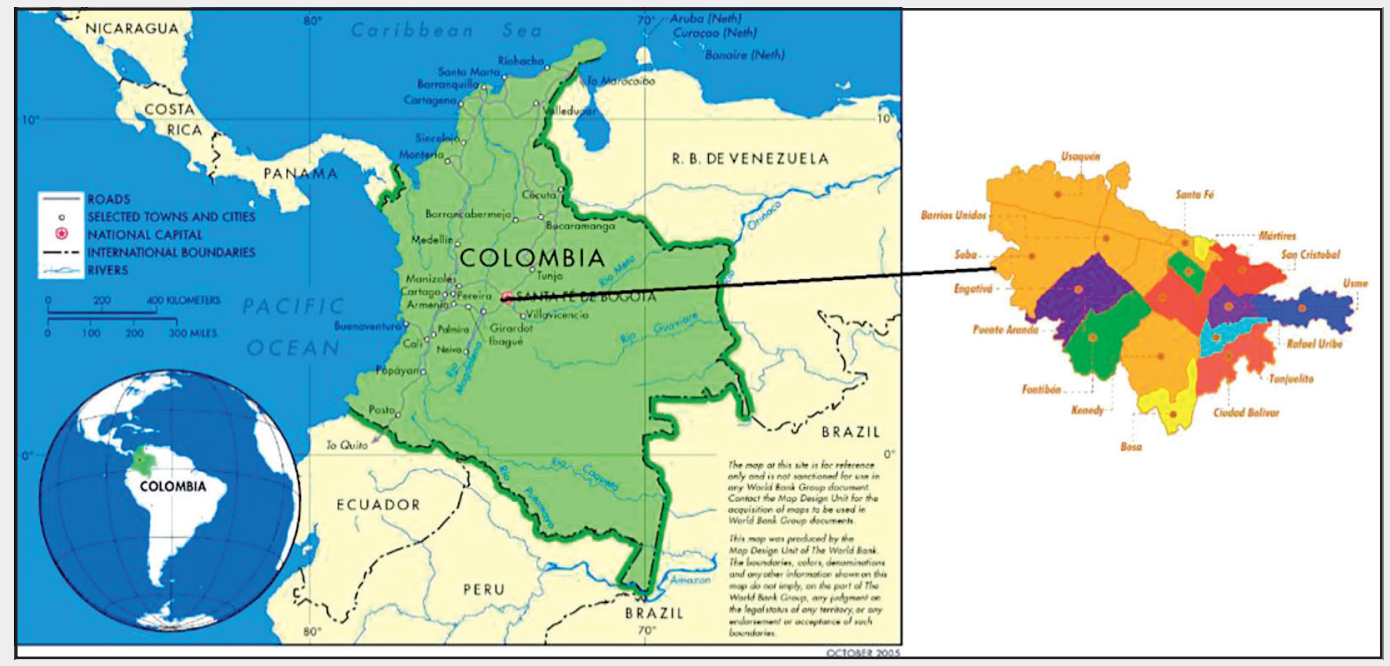

Enero - Junio 2016

ISSN 0122-820X

E-ISSN 2422-5053

Figura 1. Ubicación del sitio de estudio en la ciudad de Bogotá, Colombia. Fuente: Mapa Interactivo, 2014 [11] 
No. 1

Enero - Junio 2016 ISSN 0122-820X E-ISSN 2422-5053 PP: 6-15

10 en el formato de Residuos Hospitalarios $\mathrm{RH} 1$, formato establecido para registrar los Residuos Hospitalarios y Similares generados en un mes [12], este registro es producto de los pesajes diarios realizados en las 11 áreas generadoras de residuos en la IPS CAMI VISTA HERMOSA: laboratorio clínico, odontología, hospitalización, urgencias, medicina general, ginecología, depósito de medicamentos, rayos $\mathrm{x}$, atención de partos, sala de enfermedades respiratorias agudas (ERA) y vacunación, donde se estableció la medición, de cada uno de los pesos $(\mathrm{Kg})$, alcanzados en las bolsas totales, correspondientes a cada tipo de residuos generados en las áreas mencionadas.

\subsection{Diagnóstico del manejo de residuos en la IPS}

Para la determinación de la gestión de los residuos generados en la IPS, se realizó como primer paso un diagnóstico de las condiciones actuales de manejo de los residuos, donde se efectuó una revisión de los documentos existentes que soportan el trabajo realizado hasta el momento por parte del comité de gestión ambiental de la IPS.

Se tomó como línea base la información que la IPS tenía desde el año 2005, en cuanto a la gestión integral de sus residuos, coordinando las actividades de segregación, recolección, transporte, almacenamiento y disposición final de las diferentes clases de residuos que se generan en cada una de las áreas de la IPS (Figura 2).

Se realizó el reconocimiento general del centro y una inspección por cada área de las instalaciones, observando los aspectos relacionados con impacto ambiental $\mathrm{y}$ condiciones sanitarias presentes e identificando las condiciones actuales, en cuanto a:

- Características de la infraestructura.

- Clasificación de residuos.
- Tipo y localización de recipientes empleados para la disposición de residuos.

- Movimiento interno de residuos.

- Área de disposición central.

- Tratamiento y disposición final de los residuos.

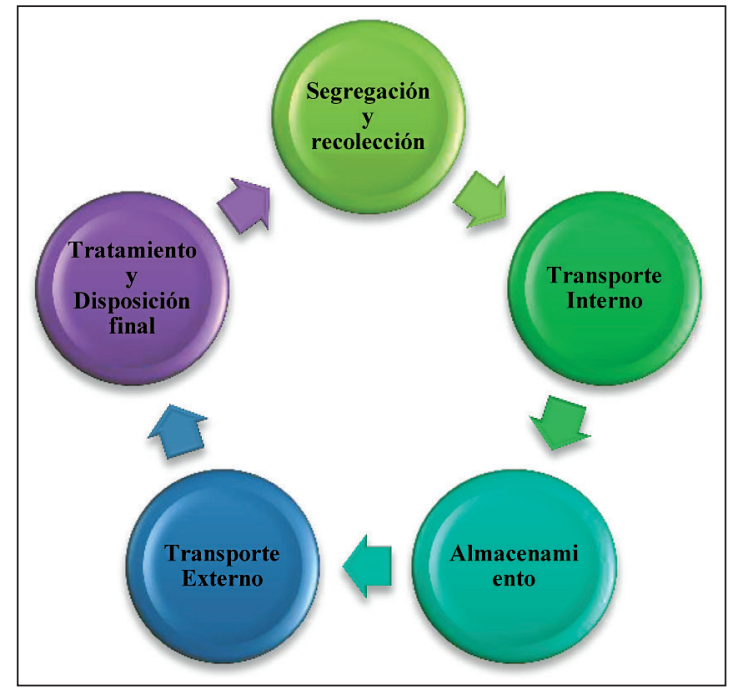

Figura 2. Sistema de gestión interno y externo de residuos. Fuente: Autor.

\section{Resultados y análisis}

Se determinó que en la IPS CAMI Vista Hermosa, no se implementan adecuadamente los procesos de segregación, recolección, manipulación y disposición final de los residuos hospitalarios y similares, originando impactos ambientales y riesgos a la salud pública, como punción por segregación incontrolada y contaminación cruzada con microrganismos presentes en el medio o en residuos propensos a la descomposición como biosanitarios y anatomopatológicos. Las infecciones nosocomiales tienen su origen en el sistema sanitario de los hospitales, capaz de albergar lo que estos conocen como flora exógena sujeta al entorno inanimado y que está compuesta principalmente por bacterias de los géneros, Staphylococcus, Escherichia, Klebsiella, Pseudomonas, Candida y Serratia [13].

En la IPS CAMI Vista Hermosa, se generan residuos de dos tipos, no peligrosos y 
peligrosos de acuerdo a los lineamientos dados por la Resolución 1164 de 2002 [12]. Se valoraron 11 áreas dentro de la institución incluyendo el laboratorio clínico, odontología, hospitalización y urgencias, de las cuales 9 generan residuos biosanitarios considerados como de alto riesgo biológico [14], los cuales son causantes de infecciones intrahospitalarias, que ponen en riesgo la salud de los trabajadores que manipulan de forma directa e indirecta elementos e insumos para la prestación de los servicios médicos.

La tabla I, consolida las áreas correspondientes al servicio asistencial, con el respectivo residuo generado, donde se evidencia que en la mayoría de ellas se generan residuos peligrosos y no peligrosos.

Tabla I. Caracterización Cualitativa

\begin{tabular}{|l|l|}
\hline \multicolumn{1}{|c|}{ FUENTE } & \multicolumn{1}{c|}{ TIPO DE RESIDUO GENERADO } \\
\hline Laboratorio Clínico & Biosanitarios, Cortopunzantes, Reactivos, Ordinarios, Reciclables y Anatomopatológicos \\
\hline Odontología & Biosanitarios, Cortopunzantes, Fármacos, Metales Pesados, Ordinarios, Reciclables y Anatomopatológicos \\
\hline Medicina General & Biosanitarios, Ordinarios y Reciclables \\
\hline Ginecología & Biosanitarios, Ordinarios y Reciclables \\
\hline Depósito de medicamentos & Fármacos, Cortopunzantes, Ordinarios y Reciclables \\
\hline Hospitalización & Biosanitarios, Cortopunzantes, Químicos, Ordinarios y Reciclables \\
\hline Rayos X & Químicos, Reciclables \\
\hline Urgencias & Biosanitarios, Cortopunzantes, Ordinarios, Químicos y Reciclables \\
\hline Atención de partos & Biosanitarios, Cortopunzantes, Ordinarios Reciclables y Anatomopatológicos \\
\hline Sala ERA & Biosanitarios, Cortopunzantes, Ordinarios y Reciclables. \\
\hline Vacunación & Biosanitarios, Cortopunzantes, Ordinarios y Reciclables. \\
\hline
\end{tabular}

Fuente: Autor

Los residuos peligrosos más abundantes son los biosanitarios (15907,91 Kg/año) (Grafico 4), anatomopatologicos $(1117,86 \mathrm{Kg} / \mathrm{año})$ y cortopunzantes (1010,4 Kg/año) (Tabla II), a la vez son los que más riesgos podrían presentar por contaminación con microrganismos y transmisión de enfermedades nosocomiales, a través de punción, cortes, contacto con la piel o mucosas y el aire.

Tabla II. Registro Cuantitativo Formato RH1

\begin{tabular}{|c|c|c|c|c|c|c|c|c|c|c|}
\hline \multicolumn{11}{|c|}{ TIPO DE RESIDUOS } \\
\hline \multirow{3}{*}{ MES } & \multicolumn{2}{|c|}{$\begin{array}{l}\text { RESIDUOS NO } \\
\text { PELIGROSOS }\end{array}$} & \multicolumn{6}{|c|}{ RESUIDOS PELIGROSOS } & \multirow{3}{*}{$\begin{array}{c}\text { TOTAL } \\
\text { RESIDUOS } \\
\text { MES (Kg) }\end{array}$} & \multirow[b]{3}{*}{$\begin{array}{l}\text { OBSERVA- } \\
\text { CIONES }\end{array}$} \\
\hline & \multirow[b]{2}{*}{$\begin{array}{l}\text { RECICLA- } \\
\text { BLES (Kg) }\end{array}$} & \multirow[b]{2}{*}{$\begin{array}{l}\text { ORDINA- } \\
\text { RIOS (Kg) }\end{array}$} & \multicolumn{3}{|c|}{ INFECCIOSOS O DE RIESGO BIOLOGICO } & \multicolumn{3}{|c|}{ QUIMICOS } & & \\
\hline & & & $\begin{array}{l}\text { BIOSANITA- } \\
\text { RIOS (Kg) }\end{array}$ & $\begin{array}{l}\text { ANATOMO } \\
\text { PATOLOGICOS } \\
\quad \text { (Kg) }\end{array}$ & $\begin{array}{l}\text { CORTO } \\
\text { PUNZAN- } \\
\text { TES } \\
\text { (Kg) }\end{array}$ & $\begin{array}{c}\text { FARMACOS } \\
(\mathrm{Kg})\end{array}$ & $\begin{array}{c}\text { METALES } \\
\text { PESADOS } \\
(\mathrm{Kg})\end{array}$ & $\begin{array}{l}\text { REACTIVOS } \\
\text { (Kg) }\end{array}$ & & \\
\hline ENER & 451,6 & 1451 & 1042 & 52,7 & 110,6 & 98, & 0,9 & 2,2 & 3209,4 & \\
\hline FEB & 637,35 & 1204 & 1161 & 86,7 & 43,5 & 26,3 & 0,2 & 2,5 & 3161,55 & \\
\hline MAR & 374,95 & 1378 & 1069 & 105,4 & 99,6 & 76,5 & 0,4 & 3,2 & 3107,05 & \\
\hline $\mathrm{ABR}$ & 334,73 & 2103,5 & 1778 & 101,2 & 110 & 54,6 & 0,3 & 2,2 & 4485,03 & \\
\hline MAY & 342,58 & 2173,9 & 1525 & 131,82 & 56,26 & 49,2 & 0,25 & 3,4 & 4282,41 & \\
\hline JUN & 427,19 & 1844,1 & 1507,71 & 75,9 & 88,1 & 73,56 & 0,22 & 2,3 & 4019,08 & \\
\hline JUL & 387,77 & 1864,2 & 1433,72 & 125,8 & 57,1 & 80,4 & 0,45 & 2,5 & 3951,94 & \\
\hline AGO & 358,75 & 1713,2 & 1234 & 48,2 & 111,34 & 53,5 & 0,2 & 3,2 & 3522,39 & \\
\hline SEP & 455,98 & 1687,65 & 1505,23 & 83,6 & 77,8 & 62,5 & 0,25 & 2,6 & 3875,61 & \\
\hline OCT & 350,39 & 1459,45 & 1354 & 110,74 & 77,7 & 50,7 & 0,13 & 3 & 3406,11 & \\
\hline NOV & 385,79 & 1344,15 & 985,8 & 113 & 91,6 & 49,1 & 0,25 & 2,3 & 2971,99 & \\
\hline DIC & 372,7 & 1559 & 1312,45 & 82,8 & 86,8 & 41,8 & 0,1 & 2,7 & 3458,35 & \\
\hline TOTAL & 4879,78 & 19782,15 & 15907,91 & 1117,86 & 1010,4 & 716,56 & 4,15 & 32,1 & 43450,91 & \\
\hline Final & & & & & & & & & 43,45091 & \\
\hline
\end{tabular}

Fuente: Formato RH1. CAMI, Vista Hermosa. I Nivel ESE, 2008 
No. 1

Enero - Junio 2016 ISSN 0122-820X

E-ISSN 2422-5053

PP: 6-15
Los datos observados en el grafico 1, representan la totalidad de residuos de tipo peligroso generados en el centro asistencial por mes. Los residuos infecciosos de riesgo biológico (IRB) y químicos (QCOS) de tipo fármacos, metales pesados y reactivos, de estos se evidencian que el mayor porcentaje de generación de residuos se presentan en los meses de Abril, Mayo y Junio, volviendo a aumentar en los meses de Septiembre, Octubre y Diciembre, lo cual está determinado en parte por la presencia de enfermedades respiratorias en niños y adultos, cuyo incremento se asocia a las épocas de lluvia en Bogotá, generando de esta manera picos epidemiológicos [15]. Es así que se establece que por presentarse aumento en la atención de los servicios de salud, en referencia a los picos respiratorios y otras sintomatologías, se aumenta el uso de los insumos biomédicos generando de esta forma un aumento de los residuos de todo tipo, principalmente los de alta peligrosidad como son los residuos Biosanitarios, Fármacos, Cortopunzantes y Anatomopatologicos [16].

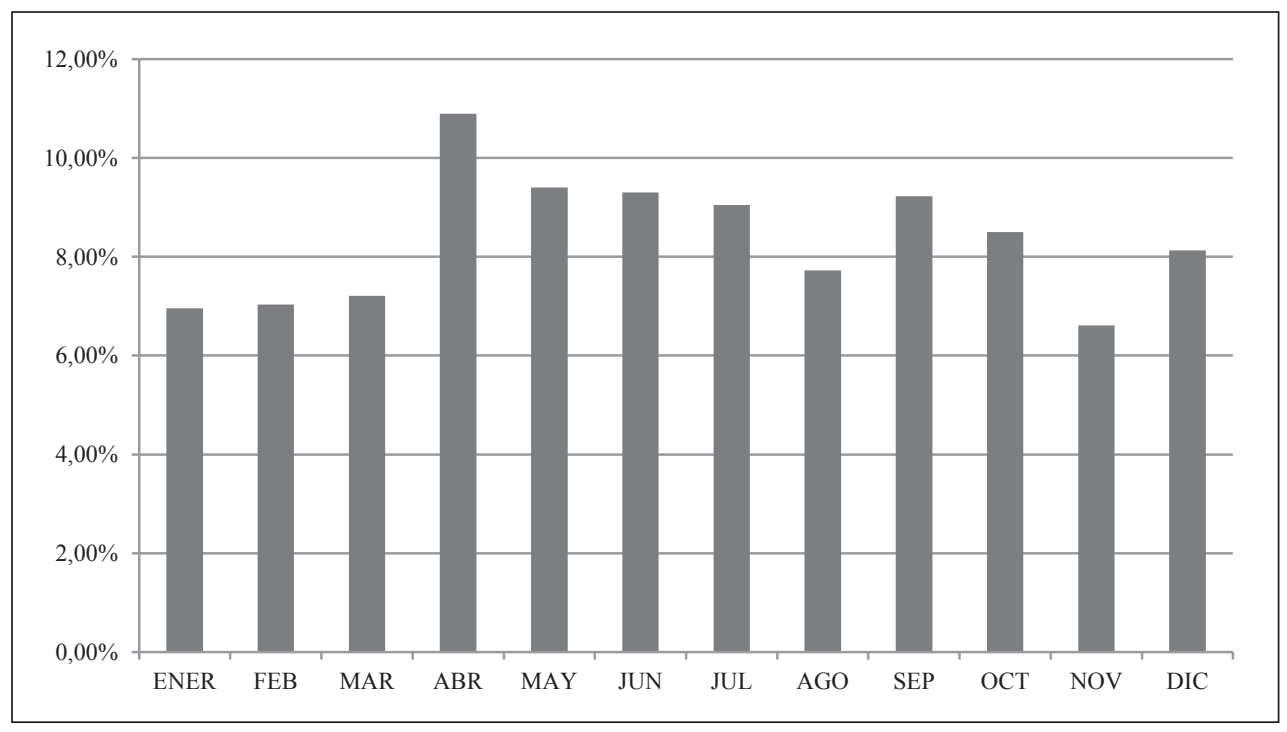

Grafico 1. Porcentajes de generación mensual de residuos peligrosos. Fuente: Modificado del Formato RH1. CAMI, Vista Hermosa. I Nivel ESE.2008

Otro aspecto que se pudo observar en la gestión de los residuos hospitalarios generados en los distintos espacios que conforman la asistencia médica de la IPS (Grafico 2), es la segregación inadecuada por parte del personal administrativo, operativo y los usuarios, que no saben en este momento como desarrollar la separación de estos residuos de una forma adecuada [5], tal es el caso de los residuos ordinarios y biosanitarios. Se encontraron residuos de otro tipo en bolsas de color rojo que indican peligrosidad, lo que limito el retiro de estos, debido al riesgo a que se ven sometidos los operarios de la institución por la contaminación.
Se establecieron lineamientos, para implementar procesos de segregación, recolección, manipulación y disposición final de forma eficiente, determinándolos en los manuales como el plan para la gestión de residuos hospitalarios y similares PGIRH'S y la consolidación del manual de bioseguridad en áreas de alto riesgo, dando como resultado la protección del ambiente y riesgos a la salud pública a través de la formulación y puesta en marcha de los distintos manuales mencionados. Los procesos de monitoreo diario, dieron herramientas para la realización de una segregación adecuada, y de esta manera enfocar la producción real en el tiempo. La 


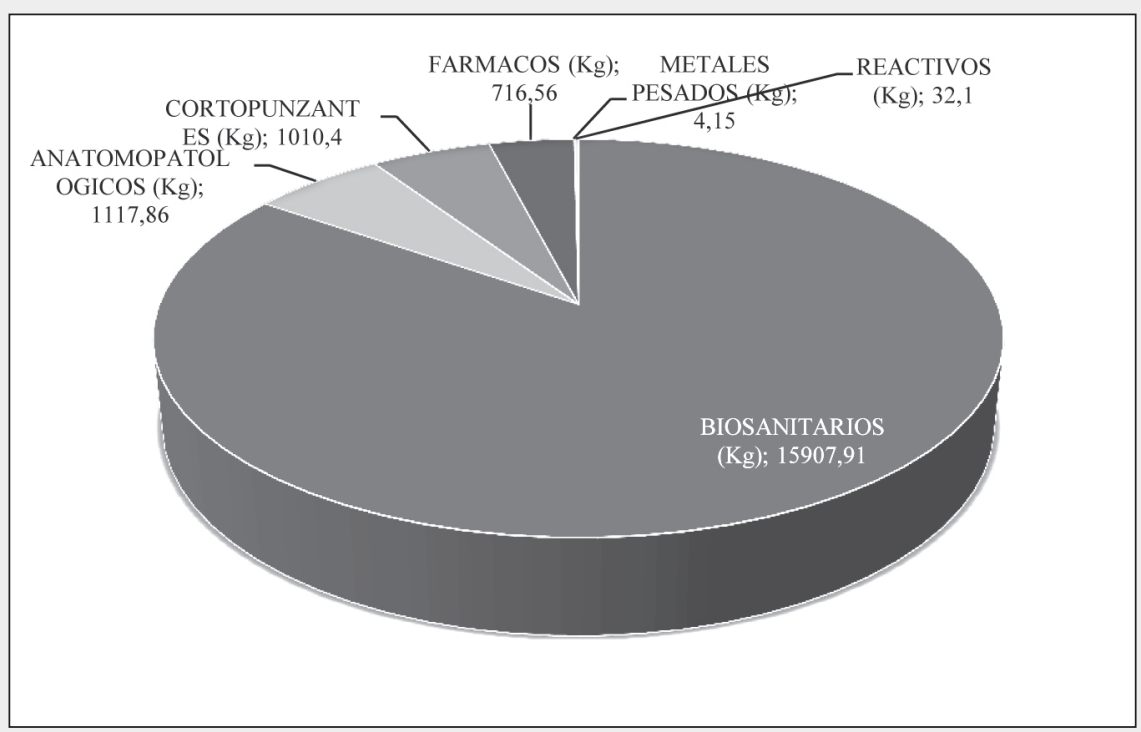

Enero - Junio 2016

recolección de información plasmada en el formato $\mathrm{RH} 1$, de acuerdo a caracterización cualitativa y cuantitativa, sirvió para reorganizar los procesos internos, los cuales son claves para tener una gestión interna eficiente de residuos, permitiendo de esta manera tener un control de la contaminación generada en cada área, los impactos generados, lo que sirvió para mitigar el riesgo ocupacional al que se encontraban inmersos el personal asistencial del hospital.

Finalmente con lo evidenciado en el proceso de investigación, se determinó desarrollar con el personal que integró el comité de gestión ambiental y sanitario del hospital, una herramienta que pudiera llegar a todo el personal asistencial, de apoyo y administrativo, relacionado a los indicadores de gestión, específicamente a la segregación, disposición de residuos y uso adecuado elementos cortopunzantes, en la herramienta creada denominada la escalera, los integrantes de cada una de las áreas asistenciales y administrativas participaban por puntajes dados a quienes mejor dominaran los temas que dan pie para el auto cuidado y el cuidado de los demás en gestión del riesgo ocupacional y cuidado al medio ambiente, logran de esta manera, iniciar un proceso de actualización y mejora en la gestión dada a los residuos hospitalarios y similares.

\section{Conclusiones}

De acuerdo a los procedimientos realizados dentro del proceso de determinación del estado de los residuos en la IPS en el periodo inicial de la investigación, se evidenció que en esta no tiene estipulado un Plan de Gestión Integral de Residuos Hospitalarios y Similares, que oriente a la gestión integral de estos, generando un cumplimiento normativo en una baja escala, los residuos son segregados de forma inadecuada $\mathrm{y}$ no se llevan los registros cualitativos y cuantitativos de la producción de residuos al interior de la institución, lo que no garantiza el cumplimiento de los lineamientos legales estipulados por la normatividad actual.

De acuerdo con la investigación realizada, se determinó el tipo de contaminación y riesgos inminentes que están presentes en la institución de acuerdo a los procedimientos aquí ejecutados. Se establecieron lineamientos, para implementar procesos de segregación, recolección, manipulación y disposición final 
No. 1

Enero - Junio 2016 ISSN 0122-820X E-ISSN 2422-5053 PP: 6-15 de forma eficiente, dando como resultado la protección del ambiente y riesgos a la salud pública a través de la formulación y puesta en marcha de un Plan de Gestión de Residuos Hospitalarios y Similares. Los procesos de monitoreo diario, dieron herramientas para la realización de una segregación adecuada, y de esta manera enfocar la producción real en el tiempo. La recolección de información plasmada en el formato RH1, de acuerdo a caracterización cualitativa y cuantitativa, sirvió para reorganizar los procesos internos, los cuales son claves para tener una gestión interna eficiente de residuos.

Los riesgos a los cuales se encuentran expuestos los usuarios, personal médico asistencial, de servicios generales y gestor externo, son los generados por una segregación inadecuada de los residuos, los cuales alteran la seguridad, la salud pública y el medio ambiente, entre los más relevantes se tienen los siguientes:

- Punción por segregación incontrolada, lo que puede generar infecciones y enfermedades profesionales e intrahospitalaria.

- Contaminación cruzada por segregación inadecuada, lo que maximiza el riesgo de adquisición de enfermedades nosocomiales a las cuales son vulnerables toda la población de la IPS, en especial el personal hospitalizado.

- Contaminación al medio ambiente, por una gestión deficiente de los residuos, ya que estos después de ser dispuestos en los recipientes establecidos para cada tipo de residuo y al ser depositados de una forma incorrecta "Segregación Inadecuada", no pueden ser separados, debido al alto riesgo que tiene dicha manipulación, así mismo al recibir un tratamiento por el gestor externo estos van a contaminar el ambiente.
Así mismo al establecerse los contaminantes y riesgos a que se encuentran inmersos el medio ambiente y la salud pública, se determinaron estrategias para controlar la operatividad y gestión de los residuos hospitalarios y similares y las normas de bioseguridad a desarrollarse en cada una de las áreas presentes en la IPS, como fue la elaboración del PGIRH'S y el respectivo manual de Bioseguridad, lo que brindo un aporte mancomunado en el desarrollo de cumplimiento de estándares normativos documentados en la Resolución 1164 de 2002 y específicamente el desarrollo de estrategias de autocuidado en esta, lo que evidencio en un mejoramiento a la gestión ambiental, una mejor segregación de los residuos y por ende una estrategia eficiente de protección a los funcionarios del centro asistencial.

\section{Referencias}

[1] Presidenciadela RepúblicadeColombia. (2000, diciembre 22). Decreto 2676 de 2000. [en línea]. Disponible en: http:// www.alcaldiabogota.gov.co/sisjur/ normas/Norma1.jsp?i=11531.

[2] Y. Quinto-Mosquera, L. M. JaramilloPérez, and J. A. Cardona-Arias. "Conocimientos y prácticas de los trabajadores de un hospital sobre el manejo de residuos hospitalarios, Chocó, Colombia, 2012". MéD. UIS, vol. 26, no. 1, pp. 9-20, 2013.

[3] R. A. Gómez Montoya, A. Zuluaga Mazo, and A. A. Correa Espinal. "Propuesta de sistema de logística inversa para el sector hospitalario: un enfoque teórico y práctico en Colombia”. Revista Ingenierias USBmed, vol. 5, no. 1, pp.35-52, 2015.

[4] A.M.Salas Bocanegra, and S.M.Vanegas Caballero. Guía de plan de manejo integral de residuos hospitalarios para instituciones prestadoras de servicios de salud de nivel II y III de complejidad. 
Revista Gestión Integral en Ingeniería Neogranadina, Universidad Militar Nueva Granada, 2010.

[5] A. Cantanhede. "La gestión y tratamiento de los residuos generados en los centros de atención de salud". Repertorio Científico, vol. 5, no. 6-7, pp.13-18, 1999.

[6] Calvo, Y., and Morales, N. D. "Diseño del plan de gestión integral de residuos sólidos hospitalarios y similares componente interno - en la E.S.E. Hospital Cumbal", trabajo de grado. Universidad Tecnologica de Pereira, Pereira, Colombia, 2011.

[7] Y. T. Hernández Peña. "El ordenamiento territorial y su construcción social en Colombia: ¿un instrumento para el desarrollo sustentable?". Cuadernos de Geografia, no. 19, pp. 97-109, 2010.

[8] F. M. Lynn, G. J. Busemberg. "Citizen advisory committee and environmental policy: what we know, what's left to discover". Risk Analysis, vol. 15, no. 2, pp. 147-162, 1995.

[9] S. G. Hadden. "Public perception of hazardous waste". Risk Analysis, vol. 11, no. 1, pp. 47-57, 1991.

[10] A. Neveu and P. Matus. "Residuos hospitalarios peligrosos en un centro de alta complejidad". Revista médica de Chile, vol. 135, no. 7, pp. 885-895, 2007.

[11] MapaInteractivo. Mapa de Bogotá. [en línea]. Disponible en: http://www. mapainteractivo.net/fotos/mapa-debogota.html. (13 de Mayo de 2014).

[12] Instituto Nacional de Salud. (Mayo de 2010). Manual de Gestión Integral de Residuos. [en línea]. Disponible en: http://www.ins.gov.co/lineas-deaccion/Red-Nacional-Laboratorios/
Documentos $\% 20$ de $\% 20$ inters $\% 20$ SRNL/PGIRH\%20INS.pdf.

[13]

M. Pujol and E. Limón. "Epidemiología general de las infecciones nosocomiales. Sistemas y programas de vigilancia". Enfermedades Infecciosas y Microbiología Clínica, vol. 31, no. 2, pp. 108-113, 2013.

[14] M. S. Hossain, A. Santhanam, N.N. Norulaini, and A. M. Omar. "Clinical solid waste management practices and its impact on human health and environment-A review". Waste management, vol. 31, no. 4, pp. 754766, 2011.

[15] N. Restrepo Centeno. "Infección respiratoria aguda en niños. un problema de salud pública no resuelto". Rev.Medica.Sanitas, vol. 16, no. 2, pp. 56-59, 2013.

[16] M. I. Pérez Giraldo, and A. M. Alzate. "Manejo de los residuos hospitalarios en la ESE Hospital Santa Mónica, Dosquebradas Risaralda", tesis Esp. Universidad Libre de Pereira, Pereira, Colombia, 2013. 